\title{
THE MIXED BVP FOR THE SECOND ORDER NONLINEAR ORDINARY DIFFERENTIAL EQUATION AT RESONANCE
}

\author{
SULKHAN MUKHIGULASHVILI
}

Received 25 November, 2015

Abstract. Efficient sufficient conditions are established for the solvability of the mixed problem

$$
u^{\prime \prime}(t)=p(t) u(t)+f(t, u(t))+h(t), \quad u(a)=0, \quad u^{\prime}(b)=0,
$$

where $h, p \in L([a, b] ; R)$ and $f \in K([a, b] \times R ; R)$, in the case where the homogeneous linear problem $w^{\prime \prime}(t)=p(t) w(t), \quad w(a)=0, w^{\prime}(b)=0$ has nontrivial solutions.

2010 Mathematics Subject Classification: 34B05; 34B10; 34B15

Keywords: nonlinear ordinary differential equation, mixed problem at resonance

\section{INTRODUCTION}

Consider on the set $I=[a, b]$ the second order nonlinear ordinary differential equation

$$
u^{\prime \prime}(t)=p(t) u(t)+f(t, u(t))+h(t) \quad \text { for } \quad t \in I
$$

with the boundary conditions

$$
u(a)=0, \quad u^{\prime}(b)=0,
$$

where $h, p \in L(I ; R)$ and $f \in K(I \times R ; R)$. By a solution of problem (1.1), (1.2) we understand a function $u \in \widetilde{C}^{\prime}(I, R)$, which satisfies equation (1.1) almost everywhere on $I$ and satisfies conditions (1.2).

Along with (1.1), (1.2) we consider the homogeneous problem

$$
\begin{gathered}
w^{\prime \prime}(t)=p(t) w(t) \quad \text { for } \quad t \in I, \\
w(a)=0, \quad w^{\prime}(b)=0 .
\end{gathered}
$$

At present, the foundations of the general theory of two-point boundary value problems are already laid and problems of this type are studied by many authors and investigated in detail (see, for instance, [3,4,10,11,13,14] and references therein). On the other hand, in all of these works, only the non-resonance case is considered. An analysis of the available literature shows that, in contrast to the Dirichlet problem, the case where the problem (1.3), (1.4) has nontrivial solutions is practically unstudied. It should be noted that, in the majority of works on this subject, the Dirichlet 
boundary value problem for the second order ordinary differential equation with the corresponding homogeneous problem possessing a nontrivial solution studied in the case where the first coefficient of the homogeneous linear problem is a constant and, more precisely, only in the simplest case where this constant is the first eigenvalue of the homogeneous linear problem (see, for instance, $[1,2,4-8,10,15]$ and references therein). In [16], we developed a technique which allowed us to establish efficient sufficient conditions (Landesman-Lazer's type conditions) for the solvability of Dirichlet $\mathrm{BVP}$ for second order ODE in the case where the first coefficient of the homogeneous linear equation is a Lebesgue integrable function (not necessarily constant) and no information is assumed on the number of zeros of the solution. (In particular, if the first coefficient in homogeneous linear equation is constant, we are able to study the cases where this constant not necessarily coincides with the first eigenvalue of the corresponding homogeneous linear problem). The theorems proved there significantly generalize and improve a number of previous results of other authors (see $[1,2,4,6,15])$.

In the present paper we generalize the method developed in article [16] for the Dirichlet boundary value problem, and prove Landesman-Lazer's type efficient sufficient conditions for solvability of problem (1.1), (1.2) in the case when the function $p \in L(I ; R)$ is not necessarily constant, under the assumption that the homogeneous problem (1.3), (1.4) has a nontrivial solution which may have arbitrarily many zeros in the interval $] a, b]$.

The results presented here are new and generalize Fredholm's third theorem for nonlinear ODE in the sense that the known Fredholm theorem is obtained in the special case where $f(t, x) \equiv 0$.

Throughout the paper we use the following notations:

$N$ is the set of all natural numbers. $R$ is the set of all real numbers, $R_{+}=[0,+\infty[$. $C(I ; R)$ is the Banach space of continuous functions $u: I \rightarrow R$ with the norm $\|u\|_{C}=\max \{|u(t)|: t \in I\}$.

$\widetilde{C}^{\prime}(I ; R)$ is the set of functions $u: I \rightarrow R$ which are absolutely continuous together with their first derivatives.

$L(I ; R)$ is the Banach space of the Lebesgue integrable functions $p: I \rightarrow R$ with the norm $\|p\|_{L}=\int_{a}^{b}|p(s)| d s$.

$K(I \times R ; R)$ is the set of the functions $f: I \times R \rightarrow R$ satisfying the Carathéodory conditions, i.e., $f(\cdot, x): I \rightarrow R$ is a measurable function for all $x \in R, f(t, \cdot): R \rightarrow R$ is a continuous function for almost all $t \in I$, and for every $r>0$ there exists $q_{r} \in$ $L\left(I ; R_{+}\right)$such that $|f(t, x)| \leq q_{r}(t)$ for almost all $t \in I,|x| \leq r$.

Having $w: I \rightarrow R$, we put:

$$
\begin{gathered}
\left.\left.N_{w} \stackrel{\text { def }}{=}\{t \in] a, b\right]: w(t)=0\right\}, \\
\Omega_{w}^{+} \stackrel{\text { def }}{=}\{t \in I: w(t)>0\}, \quad \Omega_{w}^{-} \stackrel{\text { def }}{=}\{t \in I: w(t)<0\},
\end{gathered}
$$


and $[w(t)]_{+}=(|w(t)|+w(t)) / 2, \quad[w(t)]_{-}=(|w(t)|-w(t)) / 2$ for $t \in I$.

Definition 1. Let $A$ be a finite (eventually empty) subset of $I$. We say that $f \in E(A)$, if $f \in K(I \times R ; R)$ and, for any measurable set $G \subseteq I$ and an arbitrary constant $r>0$, we can choose $\varepsilon>0$ such that if

$$
\int_{G}|f(s, x)| d s \neq 0 \text { for } x \geq r(x \leq-r)
$$

then

$$
\int_{G \backslash U_{\varepsilon}}|f(s, x)| d s-\int_{U_{\varepsilon}}|f(s, x)| d s \geq 0 \quad \text { for } \quad x \geq r(x \leq-r),
$$

where $U_{\varepsilon}=I \cap\left(\cup_{k=1}^{n}\right] t_{k}-\varepsilon / 2 n, t_{k}+\varepsilon / 2 n[)$ if $A=\left\{t_{1}, t_{2}, \ldots, t_{n}\right\}$, and $U_{\varepsilon}=\varnothing$ if $A=\varnothing$.

Remark 1. If $f \in K(I \times R ; R)$ then $f \in E(\varnothing)$.

Remark 2. It is clear that if $f(t, x) \stackrel{\text { def }}{\equiv} f_{0}(t) g_{0}(x)$, where $f_{0} \in L(I ; R)$ and $g_{0} \in$ $C(I ; R)$, then $f \in E(A)$ for every finite set $A \subset I$.

The example below shows that there exists a function $f \in K(I \times R ; R)$ such that $f \notin E\left(\left\{t_{1}, \ldots, t_{k}\right\}\right)$ for some points $t_{1}, \ldots, t_{k} \in I$.

Example 1. Let $f(t, x)=|t|^{-1 / 2} g(t, x)$ for $t \in[-1,0[\cup] 0,1], x \in R$, and $f(0,.) \equiv$ 0 , where $g(-t, x)=g(t, x)$ for $t \in]-1,1], x \in R$, and

$$
g(t, x)=\left\{\begin{array}{lll}
x & \text { for } & x \leq 1 / t, t>0 \\
1 / t & \text { for } \quad x>1 / t, t>0
\end{array} .\right.
$$

Then $f \in K([0,1] \times R ; R)$ and it is clear that $f \notin E(\{0\})$ because, for every $\varepsilon>0$, if $x \geq 1 / \varepsilon$ then $\int_{\varepsilon}^{1} f(s, x) d s-\int_{0}^{\varepsilon} f(s, x) d s=4\left(\varepsilon^{-1 / 2}-x^{1 / 2}\right)-2<0$.

\section{MAIN RESULTS}

Theorem 1. Let $i \in\{0,1\}, w$ be a nonzero solution of the problem (1.3), (1.4), $f \in E\left(N_{w}\right)$, there exists a constant $r>0$ such that the function $(-1)^{i} f$ is nondecreasing in the second argument for $|x| \geq r$,

$$
\begin{gathered}
(-1)^{i} f(t, x) \operatorname{sgn} x \geq 0 \text { for } t \in I,|x| \geq r, \\
\int_{\Omega_{w}^{+}}|f(s, r)| d s+\int_{\Omega_{w}^{-}}|f(s,-r)| d s \neq 0,
\end{gathered}
$$

and

$$
\lim _{|x| \rightarrow+\infty} \frac{1}{|x|} \int_{a}^{b}|f(s, x)| d s=0 .
$$


Then there exists $\delta>0$ such that the problem (1.1), (1.2) has at least one solution for every $h$ satisfying the condition

$$
\left|\int_{a}^{b} h(s) w(s) d s\right|<\delta\|w\|_{C} .
$$

Corollary 1. Let the assumptions of Theoreml be satisfied and

$$
\int_{a}^{b} h(s) w(s) d s=0 .
$$

Then the problem (1.1), (1.2) has at least one solution.

Example 2. From Theorem 1 it follows that the problem

$$
\begin{gathered}
u^{\prime \prime}(t)=-\lambda^{2} u(t)+\sigma|u(t)|^{\alpha} \operatorname{sgn} u(t)+h(t) \quad \text { for } 0 \leq t \leq \pi / 2 \\
u(0)=0, \quad u^{\prime}(\pi / 2)=0,
\end{gathered}
$$

with $\lambda=2 k-1(k \in N), \sigma \in\{-1,1\}$, and $\alpha \in] 0,1[$ has at least one solution if $h \in L([0, \pi / 2], R)$ is such that $\int_{0}^{\pi / 2} h(s) \sin \lambda s d s=0$.

Theorem 2. Let $i \in\{0,1\}, w$ be a nonzero solution of the problem (1.3), (1.4), $f(t, x) \stackrel{\text { def }}{\equiv} f_{0}(t) g_{0}(x)$ with $f_{0} \in L\left(I ; R_{+}\right), g_{0} \in C(R ; R)$, there exists a constant $r>0$ such that $(-1)^{i} g_{0}$ is non-decreasing for $|x| \geq r$ and

$$
(-1)^{i} g_{0}(x) \operatorname{sgn} x \geq 0 \quad \text { for } \quad|x| \geq r .
$$

Let, moreover,

$$
\left|g_{0}(r)\right| \int_{\Omega_{w}^{+}} f_{0}(s) d s+\left|g_{0}(-r)\right| \int_{\Omega_{w}^{-}} f_{0}(s) d s \neq 0
$$

and

$$
\lim _{|x| \rightarrow+\infty}\left|g_{0}(x)\right|=+\infty, \quad \lim _{|x| \rightarrow+\infty} \frac{g_{0}(x)}{x}=0 .
$$

Then, for every $h \in L(I ; R)$, the problem (1.1), (1.2) has at least one solution.

Example 3. From Theorem 2 it follows that the equation

$$
u^{\prime \prime}(t)=p_{0}(t) u(t)+p_{1}(t)|u(t)|^{\alpha} \operatorname{sgn} u(t)+h(t) \text { for } t \in I,
$$

with the conditions (1.2) has at least one solution for arbitrary $\alpha \in] 0,1\left[p_{0}, h \in\right.$ $L(I ; R)$, and such $p_{1} \in L(I ; R)$ that the condition $\sigma p_{1}(t)>0$ for $t \in I$ holds, where $\sigma \in\{-1,1\}$. 
Theorem 3. Let $i \in\{0,1\}$ and $w$ be a nonzero solution of the problem (1.3), (1.4). Let, moreover, there exist constants $r>0, \varepsilon>0$, and functions $\alpha, f^{+}, f^{-} \in$ $L\left(I ; R_{+}\right)$such that the conditions

$$
\begin{gathered}
(-1)^{i} f(t, x) \leq-f^{-}(t) \quad \text { for } \quad x \leq-r, \\
f^{+}(t) \leq(-1)^{i} f(t, x) \quad \text { for } \quad x \geq r, \\
\quad \sup \{|f(t, x)|: x \in R\} \leq \alpha(t)
\end{gathered}
$$

hold on I, and let

$$
\begin{aligned}
& -\int_{a}^{b}\left(f^{+}(s)[w(s)]_{-}+f^{-}(s)[w(s)]_{+}\right) d s+\varepsilon\|\alpha\|_{L} \\
& \leq(-1)^{i+1} \int_{a}^{b} h(s) w(s) d s \\
& \leq \int_{a}^{b}\left(f^{-}(s)[w(s)]_{-}+f^{+}(s)[w(s)]_{+}\right) d s-\varepsilon\|\alpha\|_{L} .
\end{aligned}
$$

Then the problem (1.1), (1.2) has at least one solution.

Remark 3. If $f \not \equiv 0$ then the condition $\left(2.12_{i}\right)(i=1,2)$ of Theorem 3 can be replaced by

$$
\begin{aligned}
& -\int_{a}^{b}\left(f^{+}(s)[w(s)]_{-}+f^{-}(s)[w(s)]_{+}\right) d s \\
& <(-1)^{i+1} \int_{a}^{b} h(s) w(s) d s \\
& <\int_{a}^{b}\left(f^{-}(s)[w(s)]_{-}+f^{+}(s)[w(s)]_{+}\right) d s .
\end{aligned}
$$

because from (2.15) there follows the existence of a constant $\varepsilon>0$ such that the condition $\left(2.12_{i}\right)$ is satisfied.

Remark 4. If $\tilde{f}(t)=\min \left\{f^{+}(t), f^{-}(t)\right\}$ then the condition (2.14) of Theorem 3 can be replaced by

$$
\left|\int_{a}^{b} h(s) w(s) d s\right| \leq \int_{a}^{b} \tilde{f}(s)|w(s)| d s-\varepsilon\|\alpha\|_{L} .
$$

Example 4. From Theorem 3 it follows that the equation

$$
u^{\prime \prime}(t)=-\lambda^{2} u(t)+\frac{|u(t)|^{\alpha}}{1+|u(t)|^{\alpha}} \operatorname{sgn} u(t)+h(t) \quad \text { for } \quad 0 \leq t \leq \pi / 2,
$$

where $\lambda=2 k-1(k \in N)$ and $\alpha \in] 0,+\infty[$, with the conditions (2.7) has at least one solution if $h \in L([0, \pi / 2], R)$ is such that $|h(t)|<1$ for $0 \leq t \leq \pi / 2$. 


\section{AUXILIARY PROPOSITIONS}

Let $u_{n} \in \widetilde{C}^{\prime}(I ; R),\left\|u_{n}\right\|_{C} \neq 0(n \in N), w$ be an arbitrary solution of the problem (1.3), (1.4), and $r>0$. Then, for every $n \in N$, we define:

$$
\begin{gathered}
A_{n, 1} \stackrel{\text { def }}{=}\left\{t \in I:\left|u_{n}(t)\right| \leq r\right\}, \quad A_{n, 2} \stackrel{\text { def }}{=}\left\{t \in I:\left|u_{n}(t)\right|>r\right\}, \\
B_{n, i} \stackrel{\text { def }}{=}\left\{t \in A_{n, 2}: \operatorname{sgn} u_{n}(t)=(-1)^{i-1} \operatorname{sgn} w(t)\right\} \quad(i=1,2), \\
C_{n, 1} \stackrel{\text { def }}{=}\left\{t \in A_{n, 2}:|w(t)| \geq 1 / n\right\}, \quad C_{n, 2} \stackrel{\text { def }}{=}\left\{t \in A_{n, 2}:|w(t)|<1 / n\right\}, \\
D_{n} \stackrel{\text { def }}{=}\left\{t \in I:|w(t)|>r\left\|u_{n}\right\|_{C}^{-1}+1 / 2 n\right\}, \\
A_{n, 2}^{ \pm} \stackrel{\text { def }}{=}\left\{t \in A_{n, 2}: \pm u_{n}(t)>r\right\}, \quad B_{n, i}^{ \pm} \stackrel{\text { def }}{=} A_{n, 2}^{ \pm} \cap B_{n, i}, \\
C_{n, i}^{ \pm} \stackrel{\text { def }}{=} A_{n, 2}^{ \pm} \cap C_{n, i} \quad(i=1,2), D_{n}^{ \pm} \stackrel{\text { def }}{=}\left\{t \in I: \pm w(t)>r\left\|u_{n}\right\|_{C}^{-1}+1 / 2 n\right\},
\end{gathered}
$$

From these definitions it is clear that, for any $n \in N$, we have

$$
\begin{gathered}
A_{n, 1} \cap A_{n, 2}=\varnothing, A_{n, 2}^{+} \cap A_{n, 2}^{-}=\varnothing, \quad B_{n, 1} \cap B_{n, 2}=\varnothing, C_{n, 1} \cap C_{n, 2}=\varnothing, \\
D_{n}^{+} \cap D_{n}^{-}=\varnothing, B_{n, 2}^{+} \cap B_{n, 2}^{-}=\varnothing, C_{n, i}^{+} \cap C_{n, i}^{-}=\varnothing(i=1,2),
\end{gathered}
$$

and

$$
\begin{gathered}
A_{n, 1} \cup A_{n, 2}=I, A_{n, 2}^{+} \cup A_{n, 2}^{-}=A_{n, 2}, B_{n, 1} \cup B_{n, 2}=A_{n, 2} \backslash N_{w}, \\
C_{n, 1} \cup C_{n, 2}=A_{n, 2}, B_{n, 2}^{+} \cup B_{n, 2}^{-}=B_{n, 2}, C_{n, 1}^{ \pm} \cup C_{n, 2}^{ \pm}=A_{n, 2}^{ \pm}, \\
C_{n, i}^{+} \cup C_{n, i}^{-}=C_{n, i}(i=1,2), D_{n}^{+} \cup D_{n}^{-}=D_{n} .
\end{gathered}
$$

The proofs of the following two lemmas are given in [16].

Lemma 1. Let $u_{n} \in \widetilde{C}^{\prime}(I ; R)(n \in N), r>0, w$ be an arbitrary nonzero solution of the problem (1.3), (1.4), and

$$
\begin{gathered}
\left\|u_{n}\right\|_{C} \geq 2 r n \quad \text { for } \quad n \in N, \\
\left\|v_{n}-w\right\|_{C} \leq 1 / 2 n \quad \text { for } \quad n \in N,
\end{gathered}
$$

where $v_{n}(t)=u_{n}(t)\left\|u_{n}\right\|_{C}^{-1}$. Then there exists $n_{0} \in N$ such that

$$
\begin{array}{llll}
D_{n_{0}}^{+} \subset A_{n, 2}^{+}, & D_{n_{0}}^{-} \subset A_{n, 2}^{-} \quad \text { for } & n \geq n_{0}, \\
C_{n_{0}, 1}^{+} \subset D_{n}^{+} & C_{n_{0}, 1}^{-} \subset D_{n}^{-} & \text {for } & n \geq n_{0} .
\end{array}
$$

Moreover

$$
\begin{aligned}
\lim _{n \rightarrow+\infty} \operatorname{mes} A_{n, 1}=0, & \lim _{n \rightarrow+\infty} \operatorname{mes} A_{n, 2}=\operatorname{mes} I, \\
C_{n, 1} \subset B_{n, 1}, & B_{n, 2} \subset C_{n, 2}, \\
B_{n, 2}^{+} \subset C_{n, 2}^{+}, & B_{n, 2}^{-} \subset C_{n, 2}^{-}, \\
C_{n, 1}^{+} \subset B_{n, 1}^{+}, & C_{n, 1}^{-} \subset B_{n, 1}^{-},
\end{aligned}
$$




$$
\begin{gathered}
\lim _{n \rightarrow+\infty} \operatorname{mes} C_{n, 1}=\lim _{n \rightarrow+\infty} \operatorname{mes} B_{n, 1}=\operatorname{mes} I, \\
\lim _{n \rightarrow+\infty} \operatorname{mes} C_{n, 2}=\lim _{n \rightarrow+\infty} \operatorname{mes} B_{n, 2}=0, \\
r<\left|u_{n}(t)\right| \leq\left\|u_{n}\right\|_{C} / 2 n \quad \text { for } \quad t \in B_{n, 2}, \\
\left|u_{n}(t)\right| \geq|| u_{n} \|_{C} / 2 n>r \quad \text { for } \quad t \in C_{n, 1}, \\
C_{n, 2}^{ \pm}=\left\{t \in A_{n, 2}: 0 \leq \pm w(t)<1 / n\right\}, \\
C_{n, 1}^{ \pm} \subset \Omega_{w}^{ \pm}, \quad \lim _{n \rightarrow+\infty} \operatorname{mes} C_{n, 1}^{ \pm}=\operatorname{mes} \Omega_{w}^{ \pm}
\end{gathered}
$$

Lemma 2. Let $i \in\{1,2\}, r>0, k \in N, w_{0}$ be a nonzero solution of the problem (1.3), (1.4), $N_{w_{0}}=\left\{t_{1}, \ldots, t_{k}\right\}$, the function $f_{1} \in E\left(N_{w_{0}}\right)$ be non-decreasing in the second argument for $|x| \geq r$, and

$$
f_{1}(t, x) \operatorname{sgn} x \geq 0 \quad \text { for } \quad t \in I,|x| \geq r .
$$

Then:

(a) If $G \subset I$ and

$$
\int_{G}\left|f_{1}\left(s,(-1)^{i} r\right) w_{0}(s)\right| d s \neq 0,
$$

then there exist $\delta_{0}>0$ and $\varepsilon_{1}>0$ such that

$$
\mathbb{I}\left(G, U_{\varepsilon}, x\right) \stackrel{\text { def }}{=} \int_{G \backslash U_{\varepsilon}}\left|f_{1}(s, x) w_{0}(s)\right| d s-\int_{U_{\varepsilon}}\left|f_{1}(s, x) w_{0}(s)\right| d s \geq \delta_{0}
$$

for $(-1)^{i} x \geq r$ and $0<\varepsilon \leq \varepsilon_{1}$, where $U_{\varepsilon}=I \cap\left(\cup_{j=1}^{k}\left[t_{j}-\varepsilon / 2 k, t_{j}+\varepsilon / 2 k\right]\right)$.

(b) If $u_{n} \in \widetilde{C}^{\prime}(I ; R)(n \in N), r>0, w$ is an arbitrary nonzero solution of the problem (1.3), (1.4), and the condition (3.3) holds, then there exist $\left.\left.\varepsilon_{2} \in\right] 0, \varepsilon_{1}\right]$ and $n_{0} \in N$ such that

$$
\begin{gathered}
\mathbb{I}\left(D_{n}^{+}, U_{\varepsilon}^{+}, x\right) \geq-\frac{\delta_{0}}{2} \quad \text { for } \quad x \geq r, \\
\mathbb{I}\left(D_{n}^{-}, U_{\varepsilon}^{-}, x\right) \geq-\frac{\delta_{0}}{2} \quad \text { for } \quad x \leq-r
\end{gathered}
$$

for $n \geq n_{0}$ and $0<\varepsilon \leq \varepsilon_{2}$, where $U_{\varepsilon}^{ \pm}=\left\{t \in U_{\varepsilon}: \pm w(t) \geq 0\right\}$.

Lemma 3. Let all the conditions of Lemma 1 be fulfilled and there exist $r>0$ such that the condition (3.16) holds, where $f_{1} \in K(I \times R ; R)$. Then

$$
\lim _{n \rightarrow+\infty} \inf \int_{s}^{t} f_{1}\left(\xi, u_{n}(\xi)\right) \operatorname{sgn} u_{n}(\xi) d \xi \geq 0 \quad \text { for } \quad a \leq s<t \leq b .
$$


Proof. Let

$$
\gamma_{r}^{*}(t) \stackrel{\text { def }}{=} \sup \left\{\left|f_{1}(t, x)\right|:|x| \leq r\right\} \quad \text { for } \quad t \in I .
$$

Then, according to (3.1), (3.2), and (3.16), we obtain the estimate

$$
\begin{aligned}
\int_{s}^{t} f_{1}\left(\xi, u_{n}(\xi)\right) \operatorname{sgn} u_{n}(\xi) & d \xi \\
& \geq-\int_{[s, t] \cap A_{n, 1}} \gamma_{r}^{*}(\xi) d \xi+\int_{[s, t] \cap A_{n, 2}}\left|f_{1}\left(\xi, u_{n}(\xi)\right)\right| d \xi
\end{aligned}
$$

for $a \leq s<t \leq b, n \in N$. This estimate and (3.7) imply (3.20).

Lemma 4. Let $w_{0}$ be a nonzero solution of the problem (1.3), (1.4), $r>0$, the function $f_{1} \in E\left(N_{w_{0}}\right)$ be non-decreasing in the second argument for $|x| \geq r$, condition (3.16) hold, and

$$
\int_{\Omega_{w_{0}}^{+}}\left|f_{1}(s, r)\right| d s+\int_{\Omega_{w_{0}}^{-}}\left|f_{1}(s,-r)\right| d s \neq 0 .
$$

Then there exist $\delta>0$ and $n_{1} \in N$ such that if

$$
\left|\int_{a}^{b} h_{1}(s) w_{0}(s) d s\right|<\delta\left\|w_{0}\right\|_{C}
$$

then, for every nonzero solution $w$ of the problem (1.3), (1.4), and functions $u_{n} \in$ $\widetilde{C}^{\prime}(I ; R)(n \in N)$ such that the conditions (3.3),

$$
\left|v_{n}^{(i)}(t)-w^{(i)}(t)\right| \leq 1 / 2 n \quad \text { for } t \in I, n \in N,(i=0,1)
$$

where $v_{n}(t)=u_{n}(t)\left\|u_{n}\right\|_{C}^{-1}$ for $t \in I$ and

$$
u_{n}(a)=0, u_{n}^{\prime}(b)=0
$$

are fulfilled, there exists $n_{1} \in N$ such that

$$
\mathrm{M}_{n}(w) \stackrel{\text { def }}{\equiv} \int_{a}^{b}\left(h_{1}(s)+f_{1}\left(s, u_{n}(s)\right)\right) w(s) d s \geq 0 \quad \text { for } \quad n \geq n_{1} .
$$

Proof. Without loss of generality we can assume that $\left\|w_{0}\right\|_{C}=1$. Also it is not difficult to verify that all the assumption of Lemma 1 are satisfied. Then, by the definition of the sets $B_{n, 1}, B_{n, 2}$, the conditions (3.1), (3.2), and (3.16), we obtain the estimate

$$
\int_{a}^{b} f_{1}\left(s, u_{n}(s)\right) w(s) d s \geq-\int_{A_{n, 1}} \gamma_{r}^{*}(s)|w(s)| d s+\hat{\mathbb{M}}_{n}(w),
$$

where

$$
\widehat{\mathbb{M}}_{n}(w) \stackrel{\text { def }}{=}-\int_{B_{n, 2}}\left|f_{1}\left(s, u_{n}(s)\right) w(s)\right| d s+\int_{B_{n, 1}}\left|f_{1}\left(s, u_{n}(s)\right) w(s)\right| d s .
$$


On the other hand, from the unique solvability of the Cauchy problem for the equation (1.3) it is clear that

$$
w^{\prime}(a) \neq 0, \quad w^{\prime}(b) \neq 0, \quad w^{\prime}\left(t_{i}\right) \neq 0 \quad \text { for } i=1, \ldots, k
$$

if $N_{w_{0}}=\left\{t_{1}, \ldots, t_{k}\right\}$. Now note that, for any nonzero solution $w$ of the problem (1.3), (1.4), there exists $\beta \neq 0$ such that $w(t)=\beta w_{0}(t)$. Consequently

$$
\Omega_{w}^{ \pm}=\Omega_{w_{0}}^{ \pm} \quad \text { if } \quad \beta>0 \quad \text { and } \quad \Omega_{w}^{\mp}=\Omega_{w_{0}}^{ \pm} \quad \text { if } \quad \beta<0 .
$$

Then in view of (3.15) and (3.22), there exists $n_{2} \geq n_{0}$ such that

$$
\int_{C_{n_{2}, 1}^{+}}\left|f_{1}(s, r) w_{0}(s)\right| d s \neq 0 \text { and/or } \int_{C_{n_{2}, 1}^{-}}\left|f_{1}(s,-r) w_{0}(s)\right| d s \neq 0 .
$$

From (3.30), in view of (3.6), it follows that

$$
\int_{D_{n}^{+}}\left|f_{1}(s, r) w_{0}(s)\right| d s \neq 0 \quad \text { for } \quad n \geq n_{2}
$$

and/or

$$
\int_{D_{n}^{-}}\left|f_{1}(s,-r) w_{0}(s)\right| d s \neq 0 \text { for } n \geq n_{2} .
$$

Consequently, all the assumptions of Lemma 3.2 are satisfied with $G=D_{n}^{+}$and/or $G=D_{n}^{-}$. Therefore, there exist $\left.\varepsilon_{0} \in\right] 0, \varepsilon_{2}\left[, n_{3} \geq n_{2}\right.$, and $\delta_{0}>0$ such that

$$
\begin{gathered}
\mathbb{I}\left(D_{n}^{+}, U_{\varepsilon_{0}}^{+}, x\right) \geq \delta_{0} \text { for } x \geq r, n \geq n_{3}, \\
\mathbb{I}\left(D_{n}^{-}, U_{\varepsilon_{0}}^{-}, x\right) \geq-\delta_{0} / 2 \text { for } x \leq-r, n \geq n_{3}
\end{gathered}
$$

if $\left(3.31_{1}\right)$ holds, and

$$
\begin{gathered}
\mathbb{I}\left(D_{n}^{-}, U_{\varepsilon_{0}}^{-}, x\right) \geq \delta_{0} \quad \text { for } \quad x \leq-r, n \geq n_{3}, \\
\mathbb{I}\left(D_{n}^{+}, U_{\varepsilon_{0}}^{+}, x\right) \geq-\delta_{0} / 2 \quad \text { for } \quad x \geq r, n \geq n_{3}
\end{gathered}
$$

if $\left(3.31_{2}\right)$ holds.

On the other hand, the definition of the set $U_{\varepsilon}$ and (3.14), imply that there exists $n_{4}>n_{3}$, such that

$$
C_{n, 2}^{+} \subset U_{\varepsilon_{0}}^{+}, C_{n, 2}^{-} \subset U_{\varepsilon_{0}}^{-} \text {for } n \geq n_{4} .
$$

By these inclusions, (3.2), and (3.5) we obtain

$$
C_{n, 1}^{+}=A_{n, 2}^{+} \backslash C_{n, 2}^{+} \supset D_{n_{0}}^{+} \backslash U_{\varepsilon_{0}}^{+}, C_{n, 1}^{-}=A_{n, 2}^{-} \backslash C_{n, 2}^{-} \supset D_{n_{0}}^{-} \backslash U_{\varepsilon_{0}}^{+}
$$

for $n \geq n_{4}$. First suppose that $N_{w_{0}} \neq \varnothing$ and there exists $n \geq n_{4}$ such that

$$
B_{n, 2} \neq \varnothing \text {. }
$$


Then, by taking into account that $f_{1}$ is non-decreasing in the second argument for $|x| \geq r$, (3.3), (3.12), (3.16) and the definitions of the sets $B_{n, 2}^{+}, B_{n, 2}^{-}$, we get

$$
\begin{aligned}
& \left|f_{1}\left(t, u_{n}(t)\right)\right|=f_{1}\left(t, u_{n}(t)\right) \\
& \leq f_{1}\left(t, \frac{\left\|u_{n}\right\| C}{2 n}\right)=\left|f_{1}\left(t, \frac{\left\|u_{n}\right\|_{C}}{2 n}\right)\right| \quad \text { for } t \in B_{n, 2}^{+}, \\
& \left|f_{1}\left(t, u_{n}(t)\right)\right|=-f_{1}\left(t,-u_{n}(t)\right) \\
& \leq-f_{1}\left(t,-\frac{\left\|u_{n}\right\|_{C}}{2 n}\right)=\left|f_{1}\left(t,-\frac{\left\|u_{n}\right\|_{C}}{2 n}\right)\right| \quad \text { for } t \in B_{n, 2}^{-} .
\end{aligned}
$$

Analogously, from (3.3), (3.13), (3.16), and the definitions of the sets $C_{n, 1}^{+}, C_{n, 1}^{-}$, we obtain the estimates

$$
\begin{aligned}
& \left|f_{1}\left(t, u_{n}(t)\right)\right| \geq\left|f_{1}\left(t, \frac{\left\|u_{n}\right\|_{C}}{2 n}\right)\right| \quad \text { for } \quad t \in C_{n, 1}^{+}, \\
& \left|f_{1}\left(t, u_{n}(t)\right)\right| \geq\left|f_{1}\left(t,-\frac{\| u_{n}||_{C}}{2 n}\right)\right| \quad \text { for } \quad t \in C_{n, 1}^{-} .
\end{aligned}
$$

Then from (3.1), (3.2), (3.9), (3.37) and respectively from (3.1), (3.2), (3.8), and (3.38) we have

$$
\begin{aligned}
& \int_{B_{n, 2}}\left|f_{1}\left(s, u_{n}(s)\right) w(s)\right| d s \\
& \leq \int_{B_{n, 2}^{+}}\left|f_{1}\left(s, \frac{\left\|u_{n}\right\|_{C}}{2 n}\right) w(s)\right| d s+\int_{B_{n, 2}^{-}}\left|f_{1}\left(s,-\frac{\left\|u_{n}\right\|_{C}}{2 n}\right) w(s)\right| d s \\
& \leq \int_{C_{n, 2}^{+}}\left|f_{1}\left(s, \frac{\left\|u_{n}\right\|_{C}}{2 n}\right) w(s)\right| d s+\int_{C_{n, 2}^{-}}\left|f_{1}\left(s,-\frac{\left\|u_{n}\right\|_{C}}{2 n}\right) w(s)\right| d s
\end{aligned}
$$

and respectively

$$
\begin{aligned}
& \int_{B_{n, 1}}\left|f_{1}\left(s, u_{n}(s)\right) w(s)\right| d s \geq \int_{C_{n, 1}}\left|f_{1}\left(s, u_{n}(s)\right) w(s)\right| d s \\
& \geq \int_{C_{n, 1}^{+}}\left|f_{1}\left(s, \frac{\left\|u_{n}\right\| C}{2 n}\right) w(s)\right| d s+\int_{C_{n, 1}^{-}}\left|f_{1}\left(s,-\frac{\left\|u_{n}\right\| C}{2 n}\right) w(s)\right| d s .
\end{aligned}
$$

If the condition (3.36) holds, from (3.39) and (3.40) we obtain

$$
\begin{aligned}
\frac{\hat{\mathrm{M}}_{n}(w)}{|\beta|} & \geq\left(\int_{C_{n, 1}^{+}}\left|f_{1}\left(s, \frac{\left\|u_{n}\right\|_{C}}{2 n}\right) w_{0}(s)\right| d s-\int_{C_{n, 2}^{+}}\left|f_{1}\left(s, \frac{\left\|u_{n}\right\|_{C}}{2 n}\right) w_{0}(s)\right| d s\right) \\
& +\left(\int_{C_{n, 1}^{-}}\left|f_{1}\left(s,-\frac{\left\|u_{n}\right\|_{C}}{2 n}\right) w_{0}(s)\right| d s-\int_{C_{n, 2}^{-}}\left|f_{1}\left(s,-\frac{\left\|u_{n}\right\|_{C}}{2 n}\right) w_{0}(s)\right| d s\right),
\end{aligned}
$$


whence, by (3.34) and (3.35) we get

$$
\frac{\widehat{\mathbb{M}}_{n}(w)}{|\beta|} \geq \mathbb{I}\left(D_{n}^{+}, U_{\varepsilon_{0}}^{+}, \frac{\left\|u_{n}\right\|_{C}}{2 n}\right)+\mathbb{I}\left(D_{n}^{-}, U_{\varepsilon_{0}}^{-},-\frac{\left\|u_{n}\right\|_{C}}{2 n}\right)
$$

for $n \geq n_{4}$. From (3.41) by (3.32) and (3.33) we obtain

$$
\widehat{\mathrm{M}}_{n}(w) \geq \frac{\delta_{0}|\beta|}{2} \quad \text { for } n \geq n_{4} .
$$

On the other hand, in view of (3.10), (3.16), the definition of the sets $A_{n, 2}, B_{n, 1}$, and the fact that $f_{1}$ is non-decreasing in the second argument, we obtain the estimate

$$
\begin{aligned}
\int_{B_{n, 1}}\left|f_{1}\left(s, u_{n}(s)\right) w(s)\right| d s & \geq \int_{B_{n, 1}^{+}}\left|f_{1}(s, r) w(s)\right| d s+\int_{B_{n, 1}^{-}}\left|f_{1}(s,-r) w(s)\right| d s \\
& \geq \int_{C_{n, 1}^{+}}\left|f_{1}(s, r) w(s)\right| d s+\int_{C_{n, 1}^{-}}\left|f_{1}(s,-r) w(s)\right| d s .
\end{aligned}
$$

Now suppose that there exists $n \geq n_{4}$ such that

$$
B_{n, 2}=\varnothing \text {. }
$$

Then from (3.30) and (3.43), (3.44) there follows the existence of $\delta^{*}>0$ such that $\widehat{\mathrm{M}}_{n}(w) \geq|\beta| \delta^{*}$. From this inequality and (3.42) it follows that, in both cases when (3.36) or (3.44) are fulfilled, the inequality

$$
\widehat{\mathrm{M}}_{n}(w) \geq|\beta| \delta \text { for } n \geq n_{4}
$$

holds with $\delta=\min \left\{\delta_{0} / 2, \delta^{*}\right\}$. From (3.27) by (3.7) and (3.45), we see that for any $\varepsilon \in] 0, \delta\left[\right.$ there exists $n_{1}>n_{4}$ such that

$$
\int_{a}^{b} f_{1}\left(s, u_{n}(s)\right) w(s) d s \geq|\beta|(\delta-\varepsilon) \quad \text { for } \quad n \geq n_{1},
$$

and thus

$$
\frac{\mathrm{M}_{n}(w)}{|\beta|} \geq \delta-\varepsilon-\left|\int_{a}^{b} h_{1}(s) w_{0}(s) d s\right| \text { for } n \geq n_{1} .
$$

If $N_{w_{0}}=\varnothing$ then $|w(t)|>0$ for $a<t<b$ and in view of (3.3), (3.24), (3.25) and (3.28), the condition (3.44) holds, i.e., the inequality (3.46) also holds.

Consequently since $\varepsilon>0$ is arbitrary, the inequality (3.26) from (3.46) and (3.23) follows.

Lemma 5. Let $w_{0}$ be a nonzero solution of the problem (1.3), (1.4), $r>0$, and the conditions (3.16), (3.23) hold with $f_{1}(t, x) \stackrel{\text { def }}{=} f_{0}(t) g_{1}(x)$, where $f_{0} \in L\left(I ; R_{+}\right)$ and a non-decreasing function $g_{1} \in C(R ; R)$ be such that

$$
\lim _{|x| \rightarrow+\infty}\left|g_{1}(x)\right|=+\infty .
$$


Then, for every nonzero solution $w$ of the problem (1.3), (1.4) and functions $u_{n} \in$ $\widetilde{C}^{\prime}(I ; R)(n \in N)$ fulfilling the conditions (3.3), (3.24), (3.25), the inequality (3.26) holds.

Proof. From the assumptions of our lemma it is clear that the relations (3.27)(3.35), (3.37)-(3.40) and (3.43) with $f_{1}(t, x)=f_{0}(t) g_{1}(x)$ and $w(t)=\beta w_{0}(t)(\beta \neq$ $0)$ are fulfilled.

Assuming $\int_{C_{n_{2}, 1}^{+}}\left|f_{1}(s, r) w_{0}(s)\right| d s \neq 0$, the condition (3.31 $)$ is satisfied i.e., (3.32) holds.

Now notice that from (3.15) and the equality $C_{n, 1}^{+}=\Omega_{w}^{+} \backslash\left(\Omega_{w}^{+} \backslash C_{n, 1}^{+}\right)$it follows that there exist $\varepsilon>0$ and $n_{0} \in N$ such that

$$
\int_{C_{n, 1}^{+}}\left|f_{0}(s) w_{0}(s)\right| d s \geq \int_{\Omega_{w}^{+}}\left|f_{0}(s) w_{0}(s)\right| d s-\varepsilon>0
$$

for $n \geq n_{0}$.

First consider the case when there exists $n \geq n_{4}$ such that the condition (3.44) holds. Without loss of generality we can assume that $n_{4}>n_{0}$. Then by (3.29), (3.43), (3.44) and (3.48), we obtain

$$
\widehat{\mathrm{M}}_{n}(w) \geq|\beta|\left|g_{1}(r)\right|\left(\int_{\Theta_{\beta}}\left|f_{0}(s) w_{0}(s)\right| d s-\varepsilon\right)>0,
$$

where $\Theta_{\beta}=\Omega_{w_{0}}^{+}$if $\beta>0$ and $\Theta_{\beta}=\Omega_{w_{0}}^{-}$if $\beta<0$.

Consider now the case when there exists $n \geq n_{4}$ such that (3.36) holds. From (3.3) and the definition of the set $D_{n}^{+}$it follows that $D_{n}^{+} \subset D_{n+1}^{+}$, and since $g_{1}$ is non-decreasing, from (3.32) we obtain

$$
\begin{aligned}
\mathbb{I}\left(D_{n}^{+}, U_{\varepsilon_{0}}^{+}, x\right) & =\left|g_{1}(x)\right|\left(\int_{D_{n}^{+} \backslash U_{\varepsilon_{0}}^{+}}\left|f_{0}(t) w_{0}(s)\right| d s-\int_{U_{\varepsilon_{0}}^{+}}\left|f_{0}(t) w_{0}(s)\right| d s\right) \\
& \geq\left|g_{1}(r)\right| \mu=\mathbb{I}\left(D_{n_{4}}^{+}, U_{\varepsilon_{0}}^{+}, r\right) \geq \delta_{0}
\end{aligned}
$$

for $x \geq r$, with $\mu=\int_{D_{n_{4}}^{+} \backslash U_{\varepsilon_{0}}^{+}}\left|f_{0}(s) w_{0}(s)\right| d s-\int_{U_{\varepsilon_{0}}^{+}}\left|f_{0}(s) w_{0}(s)\right| d s>0$. By the last inequality, (3.3), (3.32), and (3.41) we get

$$
\widehat{\mathbb{M}}_{n}(w) \geq|\beta|\left(\left|g_{1}(r)\right| \mu-\delta_{0} / 2\right) .
$$

Applying (3.49), (3.50) in (3.27) and taking (3.7) into account, we conclude that there exist $\varepsilon_{1}>0$ and $n_{1} \geq n_{4}$ such that

$$
|\beta|\left(\left|g_{1}(r)\right| \mu_{1}-\frac{\delta_{0}}{2}-\varepsilon_{1}\right) \leq \int_{a}^{b} f_{1}\left(s, u_{n}(s)\right) w(s) d s \quad \text { for } \quad n \geq n_{1}
$$


with $\mu_{1}=\min \left(\mu, \int_{\Omega_{w_{0}}^{+}}\left|f_{0}(s) w_{0}(s)\right| d s-\varepsilon\right)$. From (3.47) and the last inequality it is clear that, for any function $h_{1}$, we can choose $r>0$ such that the inequality (3.26) will be true. In a similar manner one can prove (3.26) in the case when $\int_{C_{n_{2}, 1}^{-}}\left|f_{1}(s, r) w_{0}(s)\right| d s \neq 0$.

Lemma 6. Let $r>0$, there exist functions $\alpha, f^{-}, f^{+} \in L\left(I, R_{+}\right)$such that the conditions

$$
\begin{array}{ccc}
f_{1}(t, x) \leq-f^{-}(t) & \text { for } & x \leq-r, \\
f^{+}(t) \leq f_{1}(t, x) & \text { for } & x \geq r
\end{array}
$$

are satisfied,

$$
\sup \left\{\left|f_{1}(t, x)\right|: x \in R\right\}=\alpha(t) \quad \text { for } \quad t \in I,
$$

and there exist a nonzero solution $w_{0}$ of the problem (1.3), (1.4) and $\varepsilon>0$ such that

$$
\begin{aligned}
& -\int_{a}^{b}\left(f^{+}(s)\left[w_{0}(s)\right]_{-}+f^{-}(s)\left[w_{0}(s)\right]_{+}\right) d s+\varepsilon\|\alpha\|_{L} \\
& \leq-\int_{a}^{b} h_{1}(s) w_{0}(s) d s \\
& \leq \int_{a}^{b}\left(f^{-}(s)\left[w_{0}(s)\right]_{-}+f^{+}(s)\left[w_{0}(s)\right]_{+}\right) d s-\varepsilon\|\alpha\|_{L} .
\end{aligned}
$$

Then, for every nonzero solution $w$ of the problem (1.3), (1.4) and functions $u_{n} \in$ $\widetilde{C}^{\prime}(I ; R)(n \in N)$ fulfilling the conditions (3.3), (3.24), and (3.25), there exists $n_{1} \in$ $N$ such that the inequality (3.26) holds.

Proof. First note that, for any nonzero solution $w$ of the problem (1.3), (1.4), there exists $\beta \neq 0$ such that $w(t)=\beta w_{0}(t)$. Moreover, it is not difficult to verify that all the assumptions of Lemma1 are satisfied for the function $w(t)=\beta w_{0}(t)$. From (3.1), (3.2), and (3.52) we get

$$
\begin{aligned}
\mathrm{M}_{n}(w) \geq & -\int_{A_{n, 1} \cup B_{n, 2}} \alpha(s)|w(s)| d s+\int_{B_{n, 1}} f_{1}\left(s, u_{n}\right) w(s) d s \\
& +\int_{a}^{b} h_{1}(s) w(s) d s .
\end{aligned}
$$

On the other hand, by the definition of the set $B_{n, 1}$ we have

$$
\operatorname{sgn} u_{n}(t)=\operatorname{sgn} w(t) \quad \text { for } t \in B_{n, 1}^{+} \cup B_{n, 1}^{-} .
$$

Hence, by (3.1), (3.2), (3.10), (3.51), and (3.55), from (3.54) we obtain the estimate

$$
\mathrm{M}_{n}(w)-\int_{a}^{b} h_{1}(s) w(s) d s
$$




$$
\begin{aligned}
& \geq-\int_{A_{n, 1} \cup B_{n, 2}} \alpha(s)|w(s)| d s++\int_{B_{n, 1}^{+}} f^{+}(s)|w(s)| d s+\int_{B_{n, 1}^{-}} f^{-}(s)|w(s)| d s \\
& \geq-\int_{A_{n, 1} \cup B_{n, 2}} \alpha(s)|w(s)| d s+\int_{C_{n, 1}^{+}} f^{+}(s)|w(s)| d s+\int_{C_{n, 1}^{-}} f^{-}(s)|w(s)| d s .
\end{aligned}
$$

Now, note that $f^{-} \equiv 0$ and $f^{+} \equiv 0$ if $f_{1}(t, x) \equiv 0$. Therefore by (3.7), (3.11), (3.15), and the inclusions $C_{n, 1}^{+} \subset \Omega_{w}^{+}, C_{n, 1}^{-} \subset \Omega_{w}^{-}$, we see that there exist $\varepsilon>0$ and $n_{1} \in N$ such that

$$
\begin{aligned}
\frac{1}{3} \varepsilon\|\alpha\|_{L} & \geq \int_{A_{n, 1} \cup B_{n, 2}} \alpha(s)\left|w_{0}(s)\right| d s \\
\int_{\Omega_{w}^{ \pm}} f^{ \pm}(s)\left|w_{0}(s)\right| d s-\frac{1}{3} \varepsilon\|\alpha\|_{L} & \leq \int_{C_{n, 1}^{ \pm}} f^{ \pm}(s)\left|w_{0}(s)\right| d s
\end{aligned}
$$

for $n \geq n_{1}$. By virtue of (3.56) and (3.57), we obtain

$$
\begin{aligned}
\frac{\mathrm{M}_{n}(w)}{|\beta|} \geq & -\varepsilon\|\alpha\|_{L}+\int_{\Omega_{w}^{+}} f^{+}(s)\left|w_{0}(s)\right| d s \\
& +\int_{\Omega_{w}^{-}} f^{-}(s)\left|w_{0}(s)\right| d s+\sigma \int_{a}^{b} h_{1}(s) w_{0}(s) d s
\end{aligned}
$$

for $n \geq n_{1}$, where $\sigma=\operatorname{sgn} \beta$. Now, by taking into account that

$$
\int_{\Omega_{w}^{ \pm}} l(s)\left|w_{0}(s)\right| d s=\int_{\Omega_{w_{0}}^{ \pm}} l(s)\left|w_{0}(s)\right| d s=\int_{a}^{b} l(s)\left[w_{0}(s)\right]_{ \pm} d s
$$

if $\beta>0$ and

$$
\int_{\Omega_{w}^{ \pm}} l(s)\left|w_{0}(s)\right| d s=\int_{\Omega_{w_{0}}^{\mp}} l(s)\left|w_{0}(s)\right| d s=\int_{a}^{b} l(s)\left[w_{0}(s)\right]_{\mp} d s
$$

if $\beta<0$ for an arbitrary $l \in L(I, R)$, from the last inequalities we get

$$
\begin{aligned}
\frac{M_{n}(w)}{|\beta|} \geq & -\varepsilon\|\alpha\|_{L}+\int_{a}^{b}\left(f^{+}(s)\left[w_{0}(s)\right]_{+}+f^{-}(s)\left[w_{0}(s)\right]_{-}\right) d s \\
& +\int_{a}^{b} h_{1}(s) w_{0}(s) d s \text { for } n \geq n_{1}
\end{aligned}
$$

if $\sigma=1$, and

$$
\begin{aligned}
\frac{\mathrm{M}_{n}(w)}{|\beta|} \geq & -\varepsilon\|\alpha\|_{L}+\int_{a}^{b}\left(f^{+}(s)\left[w_{0}(s)\right]_{-}+f^{-}(s)\left[w_{0}(s)\right]_{+}\right) d s \\
& -\int_{a}^{b} h_{1}(s) w_{0}(s) d s \text { for } n \geq n_{1}
\end{aligned}
$$


if $\sigma=-1$. From the last inequalities and (3.53) we immediately obtain (3.26).

Lemma 7. Let problem (1.3), (1.4) has the nontrivial solution. Than there exists $\varepsilon>0$ suth that the equation

$$
w^{\prime \prime}(t)=\lambda p(t) w \quad \text { for } \quad t \in I,
$$

under boundari conditions (1.4) has only the trivial solution if $\lambda \in] 1,1+\varepsilon]$.

Proof. Let $G$ be the Green's function of the boundary value problem $u^{\prime \prime}(t)=$ $0, u(a)=0, u^{\prime}(b)=0$, then problem (3.58), (1.4) is equivalent to the equation $w(t)=\lambda \Gamma(w)(t)$, where the operator $\Gamma: C(I ; R) \rightarrow C(I ; R)$ is defined by the equality $\Gamma(x)(t)=\int_{a}^{b} G(t, s) p(s) x(s) d s$. As it is well-known $\Gamma: C(I ; R) \rightarrow$ $C(I ; R)$ is a compact operator, and then for every $r>0$ the disc $|\lambda| \leq r$, contains at most finite number of characteristic values [see [9], Capitol XIII, §3, Theorem 1]. From this fact the existence of $\varepsilon>0$ such that the set $] 1,1+\varepsilon]$ does not contain the characteristic values of the equation $w(t)=\lambda \Gamma(w)(t)$, it follows. Consequently this equation, i.e., problem (3.58), (1.4) has only the trivial solution if $\lambda \in] 1,1+\varepsilon]$.

\section{PRoof of the MAin Results}

Proof of Theorem 1. Let $p_{n}(t)=\left(1+(-1)^{i} / n\right) p(t)$ and for any $n \in N$, consider the problems

$$
\begin{gathered}
u_{n}^{\prime \prime}(t)=p_{n}(t) u_{n}(t)+f\left(t, u_{n}(t)\right)+h(t) \quad \text { for } t \in I, \\
u_{n}(a)=0, \quad u_{n}^{\prime}(b)=0 .
\end{gathered}
$$

and (3.58). In view of the condition (2.3) and the fact that $(-1)^{i} f(t, x)$ is nondecreasing in the second argument for $|x| \geq r$, we obtain

$$
\lim _{n \rightarrow+\infty} \frac{1}{\left\|z_{n}\right\|_{C}} \int_{a}^{b}\left|f\left(s, z_{n}(s)\right)\right| d s=0
$$

for an arbitrary sequence $z_{n} \in C(I ; R)$ with $\lim _{n \rightarrow+\infty}\left\|z_{n}\right\|_{C}=+\infty$. Moreover, in view of Lemma 7, the problem (3.58) has only the zero solution for every $n \geq n_{0}$. Therefore, as it is well-known (see [12], Corollary 2.1, p. 2271), from the inequality (4.3) it follows that the problems (4.1), (4.2) has at least one solution, suppose $u_{n}$.

Assume that

$$
\lim _{n \rightarrow+\infty}\left\|u_{n}\right\|_{C}=+\infty
$$

and $v_{n}(t)=u_{n}(t)\left\|u_{n}\right\|_{C}^{-1}$, then the conditions

$$
\begin{gathered}
v_{n}(a)=0 \quad v_{n}^{\prime}(b)=0, \\
\left\|v_{n}\right\|_{C}=1
\end{gathered}
$$

are fulfilled, and

$$
\left.v_{n}^{\prime \prime}(t)=p_{n}(t) v_{n}(t)+\frac{1}{\left\|u_{n}\right\|_{C}}\left(f\left(t, u_{n}(t)\right)\right)+h(t)\right) .
$$


Hence, by the conditions (4.3) and (4.6), from (4.7) we get the existence of $r_{0}>0$ such that $\left\|v_{n}^{\prime}\right\|_{C} \leq r_{0}$. Consequently, in view of (4.6) by the Arzela-Ascoli lemma, without loss of generality we can assume that there exists a function $w \in \widetilde{C}^{\prime}(I, R)$ such that $\lim _{n \rightarrow+\infty} v_{n}^{(i)}(t)=w^{(i)}(t)(i=0,1)$ uniformly on $I$. From the last equality and (4.4) there follows the existence of an increasing sequence $\left\{\alpha_{k}\right\}_{k=1}^{+\infty}$ of a natural numbers, such that $\left\|u_{\alpha_{k}}\right\|_{C} \geq 2 r k$ and $\left\|v_{\alpha_{k}}^{(i)}-w^{(i)}\right\|_{C} \leq 1 / 2 k$ for $k \in N$. Without loss of generality we can suppose that $u_{n} \equiv u_{\alpha_{n}}$ and $v_{n} \equiv v_{\alpha_{n}}$. In this case we see that $u_{n}$ and $v_{n}$ are the solutions of the problems (4.1), (4.2) and (4.7), (4.5) respectively, and the inequalities

$$
\left\|u_{n}\right\|_{C} \geq 2 r n, \quad\left\|v_{n}^{(i)}-w^{(i)}\right\|_{C} \leq 1 / 2 n \text { for } n \in N
$$

are fulfilled.

From (4.7), by virtue of (4.5), (4.8) and (2.3), we obtain that $w$ is a solution of the problem (1.3), (1.4). Multiplying the equations (4.1) and (1.3) respectively by $w$ and $-u_{n}$, and therefore integrating their sum from $a$ to $b$, in view of conditions (4.2) and (1.4), we obtain

$$
(-1)^{i+1} \frac{\left\|u_{n}\right\|_{C}}{\alpha_{n}} \int_{a}^{b} p(s) w(s) v_{n}(s) d s=\int_{a}^{b}\left(h(s)+f\left(s, u_{n}(s)\right)\right) w(s) d s
$$

for $n \geq n_{0}$, where in view of conditions (4.8) the equality

$$
\lim _{n \rightarrow+\infty} \int_{a}^{b} p(s) w(s) v_{n}(s) d s=\int_{a}^{b} p(s) w^{2}(s) d s
$$

holds. On the other hand multiplying equation (1.3) by $w$, and therefore integrating from $a$ to $b$, in view of condition (1.4), we obtain

$$
\int_{a}^{b} p(s) w^{2}(s) d s=\int_{a}^{b} w^{\prime \prime}(s) w(s) d s=-\int_{a}^{b} w^{\prime 2}(s) d s<0,
$$

and from (4.9) by the last two relations we get

$$
(-1)^{i} \int_{a}^{b}\left(h(s)+f\left(s, u_{n}(s)\right)\right) w(s) d s>0 .
$$

for $n \in N \geq n_{0}$. Now note that, in view the conditions (2.1), (2.2), (2.4), (4.2), and (4.8), all the assumptions of Lemma 4 with $f_{1}(t, x)=(-1)^{i} f(t, x), h_{1}(t)=$ $(-1)^{i} h(t)$ are satisfied. Therefore, the inequality (3.26) is true, which contradicts (4.10). This contradiction proves that (4.4) does not hold and thus there exists $r_{1}>0$ such that $\left\|u_{n}\right\|_{C} \leq r_{1}$ for $n \in N$. Consequently, from (4.1) and (4.2) it is clear that there exists $r_{1}^{\prime}>0$ such that $\left\|u_{n}^{\prime}\right\|_{C} \leq r_{1}^{\prime} \quad$ and $\quad\left|u_{n}^{\prime \prime}(t)\right| \leq \sigma(t)$ for $t \in I, n \in N$, where $\sigma(t)=2|p(t)| r_{1}+|h(t)|+\gamma_{r_{1}}(t)$. Hence, by Arzela-Ascoli lemma, without loss of generality we can assume that there exists a function $u_{0} \in \widetilde{C}^{\prime}(I ; R)$ such that $\lim _{n \rightarrow+\infty} u_{n}^{(i)}(t)=u_{0}^{(i)}(t)(i=0,1)$ uniformly on $I$. Therefore, it follows from (4.1) and (4.2) that $u_{0}$ is a solution of the problem (1.1), (1.2). 
Proof of Theorem 2. The proof is the same as the proof of Theorem 1. The only difference is that we use Lemma 5 instead of Lemma 4.

Proof of Theorem 3. From (2.13) it is clear that, for an arbitrary sequence $z_{n} \in$ $C(I ; R)$ such that $\lim _{n \rightarrow+\infty}\left\|z_{n}\right\|_{C}=+\infty$, the equality (4.3) holds. From (4.3) and Lemma 6, analogously as in the proof of Theorem 1, we show that the problem (1.1), (1.2) has at least one solution.

\section{ACKNOWLEDGEMENT}

This paper was supported by the Czech Science Foundation. Name of the project: "Development of new methods of solving dynamic models of corporate processes management". Project No.: GA16-03796S.

\section{REFERENCES}

[1] S. Ahmad, "A resonance problem in which the nonlinearity may grow linearly," Proc. Amer. Math. Soc., vol. 92, no. 3, pp. 381-384, 1984, doi: 10.2307/2044839.

[2] M. Arias, "Existence results on the one-dimensional Dirichlet problem suggested by the piecewise linear case," Proc. Amer. Math. Soc., vol. 97, no. 1, pp. 121-127, 1986, doi: 10.2307/2046092.

[3] R. Conti, "Equazioni differenziali ordinarie quasilineari con condizioni lineari," Ann. Mat. Pura Appl. (4), vol. 57, pp. 49-61, 1962, doi: 10.1007/BF02417726.

[4] C. De Coster and P. Habets, "Upper and lower solutions in the theory of ODE boundary value problems: classical and recent results," in Non-linear analysis and boundary value problems for ordinary differential equations (Udine), ser. CISM Courses and Lectures, 1996, vol. 371, pp. 1-78.

[5] P. Drábek, "On the resonance problem with nonlinearity which has arbitrary linear growth," $J$. Math. Anal. Appl., vol. 127, no. 2, pp. 435-442, 1987, doi: 10.1016/0022-247X(87)90121-1.

[6] R. Iannacci and M. N. Nkashama, "Nonlinear boundary value problems at resonance," Nonlinear Anal., vol. 11, no. 4, pp. 455-473, 1987, doi: 10.1016/0362-546X(87)90064-2.

[7] R. Iannacci and M. N. Nkashama, "Nonlinear two-point boundary value problems at resonance without Landesman-Lazer condition," Proc. Amer. Math. Soc., vol. 106, no. 4, pp. 943-952, 1989, doi: $10.2307 / 2047278$.

[8] R. Kannan, J. J. Nieto, and M. B. Ray, "A class of nonlinear boundary value problems without Landesman-Lazer condition,” J. Math. Anal. Appl., vol. 105, no. 1, pp. 1-11, 1985, doi: 10.1016/0022-247X(85)90093-9.

[9] L. V. Kantorovich and G. P. Akilov, Functional analysis, 2nd ed. Pergamon Press, OxfordElmsford, N.Y., 1982, translated from the Russian by Howard L. Silcock.

[10] I. T. Kiguradze, "On a singular boundary value problem,” J. Math. Anal. Appl., vol. 30, pp. 475489, 1970, doi: 10.1016/0022-247X(70)90135-6.

[11] I. T. Kiguradze, Nekotorye singulyarnye kraevye zadachi dlya obyknovennykh differentsialnykh uravnenii. Izdat. Tbilis. Univ., Tbilisi, 1975.

[12] I. T. Kiguradze, "Boundary value problems for systems of ordinary differential equations," in Current problems in mathematics. Newest results, Vol. 30 (Russian), ser. Itogi Nauki i Tekhniki. Akad. Nauk SSSR, Vsesoyuz. Inst. Nauchn. i Tekhn. Inform., Moscow, 1987, pp. 3-103, 204, translated in J. Soviet Math. 43 (1988), no. 2, 2259-2339, doi: 10.1007/BF01100360.

[13] I. T. Kiguradze and B. L. Shekhter, "Singular boundary value problems for second-order ordinary differential equations," in Current problems in mathematics. Newest results, Vol. 30 (Russian), 
ser. Itogi Nauki i Tekhniki. Akad. Nauk SSSR, Vsesoyuz. Inst. Nauchn. i Tekhn. Inform., Moscow, 1987, pp. 105-201, 204, translated in J. Soviet Math. 43 (1988), no. 2, 2340-2417, doi: 10.1007/BF01100361.

[14] J. Kurzweil, "Generalized ordinary differential equations," Czechoslovak Math. J., vol. 8 (83), pp. 360-388, 1958.

[15] E. M. Landesman and A. C. Lazer, "Nonlinear perturbations of linear elliptic boundary value problems at resonance," J. Math. Mech., vol. 19, pp. 609-623, 1969/1970.

[16] S. Mukhigulashvili, "The Dirichlet BVP for the second order nonlinear ordinary differential equation at resonance," Ital. J. Pure Appl. Math., no. 28, pp. 179-206, 2011, doi: 10.1002/mana.201500247.

\section{Author's address}

\section{Sulkhan Mukhigulashvili}

Brno University of Technology, Faculty of Business and Management, Kolejni 2906/4, 61200 Brno, Czech Republic,

Ilia State University, School of Natural Sciences and Engineering, I. Chavchavadze Str. No. 32, 0179 Tbilisi, Georgia

E-mail address: smukhigegmail.com 